\title{
Variables as Resource in Hoare Logics
}

\author{
Matthew Parkinson and Richard Bornat \\ School of Computing \\ Middlesex University \\ LONDON, UK \\ mjp41@cam.ac.uk,richard@bornat.me.uk
}

\author{
Cristiano Calcagno \\ Department of Computing \\ Imperial College \\ University of London, LONDON, UK \\ ccris@doc.ic.ac.uk
}

\begin{abstract}
Hoare logic is bedevilled by complex and unmemorable side conditions on the use of variables. We define a logic free of side conditions, and show that it admits translations of proofs in Hoare logic, thereby showing that nothing is lost. Our work draws on ideas from separation logic: program variables are treated as resource and separated with $\star$, rather than as logical variables in disguise. For clarity we exclude a treatment of the heap.
\end{abstract}

\section{Introduction}

The glory of Hoare logic [8] is the variable-assignment axiom, which converts difficult semantic arguments about program state into simple syntactic substitutions. That success depends on punning program variables in commands with identically-named logical variables in assertions, but program variables are not logical variables: they have location (lvalue or lv in Strachey's classification [17], otherwise 'address') as well as value (Strachey's rvalue or rv, otherwise 'content').

The price of the pun is a proliferation of well-chosen but complex side conditions, notably on procedure-call $[9,7,6]$ and concurrency [14]. Most of those who have worked in Hoare logic find them unpleasant and unmemorable.

Consider, for example, the concurrency rule, from [14],

$$
\begin{aligned}
& \frac{\{P 1\} C 1\{Q 1\} \quad\{P 2\} C 2\{Q 2\}}{\{P 1 \wedge P 2\} C 1 \| C 2\{Q 1 \wedge Q 2\}} \\
& \left(\begin{array}{l}
\operatorname{mods}(C 1) \cap(\mathrm{FV}(P 2) \cup \mathrm{FV}(Q 2))=\varnothing, \\
\operatorname{mods}(C 2) \cap(\mathrm{FV}(P 1) \cup \mathrm{FV}(Q 1))=\varnothing
\end{array}\right)
\end{aligned}
$$

In addition to the side condition, soundness requires us to rule out data races. This demands restrictions on the programs $C 1$ and $C 2$ which are too complex to explain in this introduction.

We want a Hoare logic without side conditions which allows us to prove all the programs that the original can prove. The second part of this requirement is the hard bit. The first is trivial if we abandon the variable-assignment axiom and make assignment alter locations rather than program/logical variables. This is the approach taken by separation logic $[15,11,16]$ towards the heap. It has led to an elegant solution to the difficult problem of reasoning about mutation of heap data structures. But, if we were to follow that path to a treatment of variables, there would be specifications which we would not be able to prove but which can be proved in Hoare logic: for example,

$$
\{y=3\} x:=y \| z:=y\{x=y=z=3\}
$$

That is, we would fail the second part of our requirement. Furthermore, our specifications would involved more complicated assertions than hitherto.

Bornat has described a Hoare logic with trivial wellformedness side conditions [3] and showed by example that it could support local formal proofs of shared-variable concurrency without restrictions on the actions of programs. In this work we build upon this to define a logic which:

1. has no side conditions; and

2. accepts a translation of any Hoare logic proof.

Our work draws on ideas from separation logic: program variables are treated as resource and separated with $\star$. For clarity we exclude a treatment of the heap. Our logic can be extended to to deal with it, and this would eliminate the embarrassment of using two mechanisms to deal with separation: side conditions to deal with the stack, and $(\star)$ to deal with the heap.

\section{Variables as resource}

Before setting out the formal details, we describe some intuitive notions behind variables as resource. Hoare logic does not deal with the ownership of variables, as illustrated by the triple

$$
\{y=0\} x:=7\{y=0\}
$$


which alters $x$ but disingenuously avoids mentioning the fact. But $x$ should be mentioned, because to execute $x:=7$ there must be a variable $x$ in the stack. Furthermore, the assignment must own that variable, in the sense that no concurrent program can safely be permitted to read it. We must also know that variable $y$ cannot be altered by some other program, else the assertion $y=0$ cannot be assumed to be invariant. And then there is the matter of variable aliasing: $x$ and $y$, distinct as names and as logical variables, must name distinct program variables - that is, distinct locations in the stack.

Assignment requires total ownership, and must be exclusive. But read access need not be exclusive, and can be shared. We use the notion of permission [2] to perform the necessary resource accounting. A total permission may be split into two read permissions, which may themselves be split further, and split permissions may be recombined $\left(p \circledast p^{\prime}\right)$. Any permission at all gives read access and at the same time denies that any other program can hold a total permission.

\section{A logic of variables as resource}

The syntax of assertions $\Phi$ is

$$
\begin{aligned}
\Phi::= & E=E\left|\operatorname{emp}_{\mathbf{s}}\right| \operatorname{Own}_{p}(x)|\pi=\pi| \\
& \Phi \Rightarrow \Phi \mid \text { false }|\forall X \cdot \Phi| \Phi \star \Phi \mid \Phi \star \Phi
\end{aligned}
$$

We distinguish integer logical variables $X, Y, \ldots$, permission logical variables $p, \ldots$ and integer program variables $x, y, \ldots$ We do not quantify over the values of program variables. $E$ and $\pi$ ranges over integer and permission expressions respectively.

\subsection{Model}

Stacks $s$ are finite partial maps from program variable names to pairs of an integer and a permission. ${ }^{1}$ Interpretations $i$ are finite partial maps from logical variable names to integers.

$$
\begin{array}{lll}
s: \mathcal{S} & \stackrel{\text { def }}{=} \text { PVarNames } \rightarrow_{\text {fin }} \text { Int } \times \text { Perms } \\
i: \mathcal{R} \stackrel{\text { def }}{=} \text { LVarNames } \rightarrow_{\text {fin }} \text { Int } \cup \text { Perms }
\end{array}
$$

We use $\llbracket E \rrbracket_{(s, i)}$ for the evaluation of expressions, and $\llbracket E \rrbracket_{s}$ will do when $E$ does not contain logical variables.

We use $\iota$ to range over elements of the permissions set. We require that the set of permissions is equipped with a

${ }^{1}$ Permissions are integral to our logic. They allow shared reading and without them we would not be able to emulate Hoare logic side conditions. partial function $\circledast:$ Perms $\times$ Perms $\rightarrow$ Perms and a distinguished element $T \in$ Perms, such that (Perms, $\circledast)$ forms a partial cancellative commutative semigroup with the following properties:

$$
\begin{array}{rr}
\text { DIVISIBILITY } & \forall \iota \cdot \exists \iota^{\prime}, \iota^{\prime \prime} \cdot\left(\iota^{\prime} \circledast \iota^{\prime \prime}=\iota\right) \\
\text { TOTAL PERMISSION } & \forall \iota \cdot(\top \circledast \iota \text { is undefined }) \\
\text { NO UNIT } \forall \iota, \iota^{\prime} \cdot\left(\iota \circledast \iota^{\prime} \neq \iota\right)
\end{array}
$$

Permissions expression are taken from the following grammar:

$$
\pi::=\iota|p| \pi \circledast \pi
$$

We define a (partial) evaluation operation on permissions expressions in the obvious way.

A forcing semantics is given in table 1. $s \# s^{\prime}$ asserts that two stacks are compatible, agreeing about values where their domains intersect and not claiming too much permission; $s \star s^{\prime}$ expresses separation; $\langle a, b\rangle$ is an element of a function; $\oplus$ is function update; $\uplus$ is disjoint function extension.

$\mathrm{Own}_{\pi}(x)$ asserts ownership of a stack containing a variable called $x$ and permission $\pi$ to access it. Crucially it also asserts that this is all that the stack contains. It says nothing about the content of the variable; it is purely about the lvalue of $x$ (cf. $E \mapsto F$ in separation logic, which asserts a single-cell heap). $\mathrm{Own}_{\mathrm{T}}(x)$ asserts total permission, i.e. ownership, and $\mathrm{Own}_{-}(x)$ means $\exists p \cdot\left(\mathrm{Own}_{p}(x)\right)$. $\mathbf{e m p}_{\mathbf{s}}$ asserts the empty stack, and true holds of any stack at all. Following separation logic, $(\star)$ combines stack assertions: Own_( $x) \star \mathrm{Own}_{-}(y)$ is a two-variable stack; $\mathrm{Own}_{\pi}(x) \star \mathrm{Own}_{\pi^{\prime}}(x)$ is equivalent to $\mathrm{Own}_{\pi \circledast \pi^{\prime}}(x)$ and therefore $\mathrm{Own}_{\mathrm{\top}}(x) \star \mathrm{Own}_{\pi}(x)$ is false; Own $(x) \star$ true is a stack which contains at least the variable $x$.

Arithmetic equality and inequality imply a level of ownership but are loose about the stack in which they operate: $x=1$, for example, implicitly asserts

$$
\left(\mathrm{Own}_{-}(x) \star \text { true }\right) \wedge \operatorname{rv}(x)=1
$$

Our logic does not admit as a tautology $E \neq F \Longleftrightarrow$ $\neg(E=F) . x \neq 1$, for example, is satisfied by any stack in which there is a cell called $x$ which contains $1 ; \neg(x=1)$, on the other hand, is satisfied by the same stacks and by those (for example emps $\mathbf{s}_{\mathbf{s}}$ ) in which $x$ does not occur at all.

$$
\begin{aligned}
& \text { Definition 1. } \\
& \quad x 1_{\pi 1}, \ldots, x n_{\pi n} \Vdash P \stackrel{\text { def }}{=} \\
& \quad\left(\mathrm{Own}_{\pi 1}(x 1) \star \ldots \star \mathrm{Own}_{\pi n}(x n)\right) \wedge P
\end{aligned}
$$

\subsection{Rules}

Our programming language is the language of Hoare logic plus variable declarations 'local-in-end' and procedure declarations 'let-=-in-end'. For simplicity we 
Table 1. Forcing Semantics $\quad(s, i) \vDash \Phi$

$$
\begin{aligned}
(s, i) \vDash E 1=E 2 & \Longleftrightarrow \llbracket E 1 \rrbracket(s, i)=\llbracket E 2 \rrbracket_{(s, i)} \wedge \mathrm{FV}(E 1=E 2) \subseteq \operatorname{dom} s \cup \operatorname{dom} i \\
(s, i) \vDash \Phi \Rightarrow \Phi^{\prime} & \Longleftrightarrow((s, i) \vDash \Phi) \Rightarrow\left((s, i) \vDash \Phi^{\prime}\right) \\
(s, i) \vDash \Phi \star \Phi^{\prime} & \Longleftrightarrow \exists s 1, s 2 \cdot\left(s=s 1 \star s 2 \wedge((s 1, i) \vDash \Phi) \wedge\left((s 2, i) \vDash \Phi^{\prime}\right)\right) \\
(s, i) \vDash \Phi \star \Phi^{\prime} & \Longleftrightarrow \forall s 1 \cdot\left(s \# s 1 \wedge((s 1, i) \vDash \Phi) \Rightarrow\left((s \star s 1, i) \vDash \Phi^{\prime}\right)\right) \\
(s, i) \vDash \operatorname{Own}_{\pi}(x) & \Longleftrightarrow \llbracket \pi \rrbracket_{(s, i)} \text { is defined } \wedge s=\{\langle x,(-, \llbracket \pi \rrbracket(s, i))\} \\
(s, i) \vDash \text { emp }_{\mathrm{s}} & \Longleftrightarrow s=\{\} \\
(s, i) \vDash \text { false } & \Longleftrightarrow \text { false } \\
(s, i) \vDash \forall X \cdot \Phi & \Longleftrightarrow \forall v \cdot((s, i \oplus\langle X, v\rangle) \vDash \Phi)
\end{aligned}
$$

We encode true, $\wedge, \vee, \exists$ and $\neg$ : e.g. $A \vee B$ is $(A \Rightarrow$ false $) \Rightarrow B$.

$$
\begin{aligned}
& s \# s^{\prime} \Longleftrightarrow \forall x, v, v^{\prime}, \iota, \iota^{\prime} \cdot\left(s(x)=(v, \iota) \wedge s^{\prime}(x)=\left(v^{\prime}, \iota^{\prime}\right) \Rightarrow v=v^{\prime} \wedge \exists \iota^{\prime \prime} \cdot\left(\iota^{\prime \prime}=\iota \circledast \iota^{\prime}\right)\right) \\
& s \star s^{\prime}=\left\{\begin{array}{ll}
\langle x,(v, p)\rangle & \begin{array}{l}
\left(s(x)=(v, \iota) \wedge x \notin \operatorname{dom}\left(s^{\prime}\right)\right) \\
\vee\left(s^{\prime}(x)=(v, \iota) \wedge x \notin \operatorname{dom}(s)\right) \\
\vee\left(s(x)=\left(v, \iota^{\prime}\right) \wedge s^{\prime}(x)=\left(v, \iota^{\prime \prime}\right) \wedge \iota=\iota^{\prime} \circledast \iota^{\prime \prime}\right)
\end{array}
\end{array}\right\}, \quad \text { where } s \# s^{\prime} ; \\
& \text { undefined, }
\end{aligned}
$$

consider procedures each of which have a single call-byreference parameter $x$ and a single call-by-value parameter $y$. It would be straightforward to extend this treatment to deal with other cases. The rules of our program logic are given in table 2 . $\Gamma$ is the function context, a set of function specifications $\{\Phi\} f(x, Y)\left\{\Phi^{\prime}\right\}$, and $O$ ranges over ownership assertions $x 1_{\pi 1}, \ldots, x n_{\pi n}$.

The first assignment axiom can be used in forward reasoning. The second is a weakest pre-condition version which can be derived from the first. The if and while rules have an antecedent $\Phi \Rightarrow B=B$, which ensures that variables mentioned in $B$ are in the stack. In the let rule we give the function body $C$ total permission to access the value parameter $y$. The first function-call rule deals with reference arguments by straightforward $\alpha$-conversion. The second, an axiom, deals with value arguments, and is subtle. You might have expected to see

$$
\Gamma,\{\Phi\} f(x ; Y)\left\{\Phi^{\prime}\right\} \vdash_{v r}\{\Phi[E / Y]\} \quad f(x ; E)\left\{\Phi^{\prime}[E / Y]\right\}
$$

But suppose that $\Phi$ is $Y=3 \wedge \mathbf{e m p}_{\mathbf{s}}$ : then $\Phi$ claims no stack, but $\Phi[E / Y]$ is $E=3 \wedge \mathbf{e m p}_{\mathbf{s}}$, which is false if $E$ mentions any program variables. Or you you might have expected

$$
\left.\Gamma,\{\Phi\} f(x ; Y)\left\{\Phi^{\prime}\right\} \vdash_{v r}\{\Phi \wedge Y=E]\right\} \quad f(x ; E)\left\{\Phi^{\prime}\right\}
$$

But if $\Phi$ is $Y=3 \wedge \mathrm{Own}_{\mathrm{T}}(x)$, then the precondition $Y=$ $3 \wedge \mathrm{Own}_{\mathrm{T}}(x) \wedge Y=E$ is false if $E$ mentions any program variables other than $x$. In the axiom of table $2 \Psi$ claims the stack that $E$ claims but $\Phi$ does not, and $(\Phi \star \Psi) \wedge Y=E$ allows the procedure call to read and/or write variables that are mentioned both in $E$ and $\Phi$ as well as to be provided with a value to use in place of $Y$.

\subsection{Soundness}

An operational semantics is given in table 3. In $s \stackrel{C}{\longrightarrow}_{\rho}^{n} s^{\prime}$

- $s$ and $s^{\prime}$ are stacks;

- $C$ is a command;

- $\rho$ maps procedure names to a triple $\left(x, y, C^{\prime}\right)$ of reference-parameter name $x$, value-parameter name $y$ and command $C^{\prime}$; and

- $n$ is a recursion-depth counter.

A safe computation - the top part of the table and definition 2 - does not access stack locations that are undefined. The lower part of the table deals with unsafe computations, which access variables for which they have no permission.

Definition 2. $s \stackrel{C}{\longrightarrow}_{\rho}^{n}$ safe iff $\forall n . \neg(s \stackrel{C}{\longrightarrow} \rho$ unsafe $)$

Lemma 3. If $s \stackrel{C}{\longrightarrow}_{\rho}^{n}$ safe and $s^{\prime} \# s$ then $s \star s^{\prime} \stackrel{C}{\longrightarrow}_{\rho}^{n}$ safe

Proof. By induction on the evaluation rules.

Lemma 4 (Locality). If $s \stackrel{C}{\longrightarrow}{ }_{\rho}^{n}$ safe and $s^{\prime} \# s$ and $s \star s^{\prime} \stackrel{C}{\longrightarrow}_{\rho}^{n}$ $s 1$ then $\exists s 2 \cdot s \stackrel{C}{\longrightarrow} \underset{\rho}{n}$ s2 and $s 2 \star s^{\prime}=s 1$.

Proof. By induction on the evaluation rules.

Choice of fresh variable does not affect the reduction, and hence the semantics are deterministic with respect to the stack.

Definition $5(\leftrightarrow)$.

$$
\begin{aligned}
& ((y \leftrightarrow x) s) x \stackrel{\text { def }}{=}((x \leftrightarrow y) s) x \stackrel{\text { def }}{=} s y ; \\
& ((x \leftrightarrow y) s) z \stackrel{\text { def }}{=} s z .
\end{aligned}
$$


Table 2. Axioms and Rules $\quad \Gamma \vdash_{v r}\{\Phi\} C\{\Phi\}$

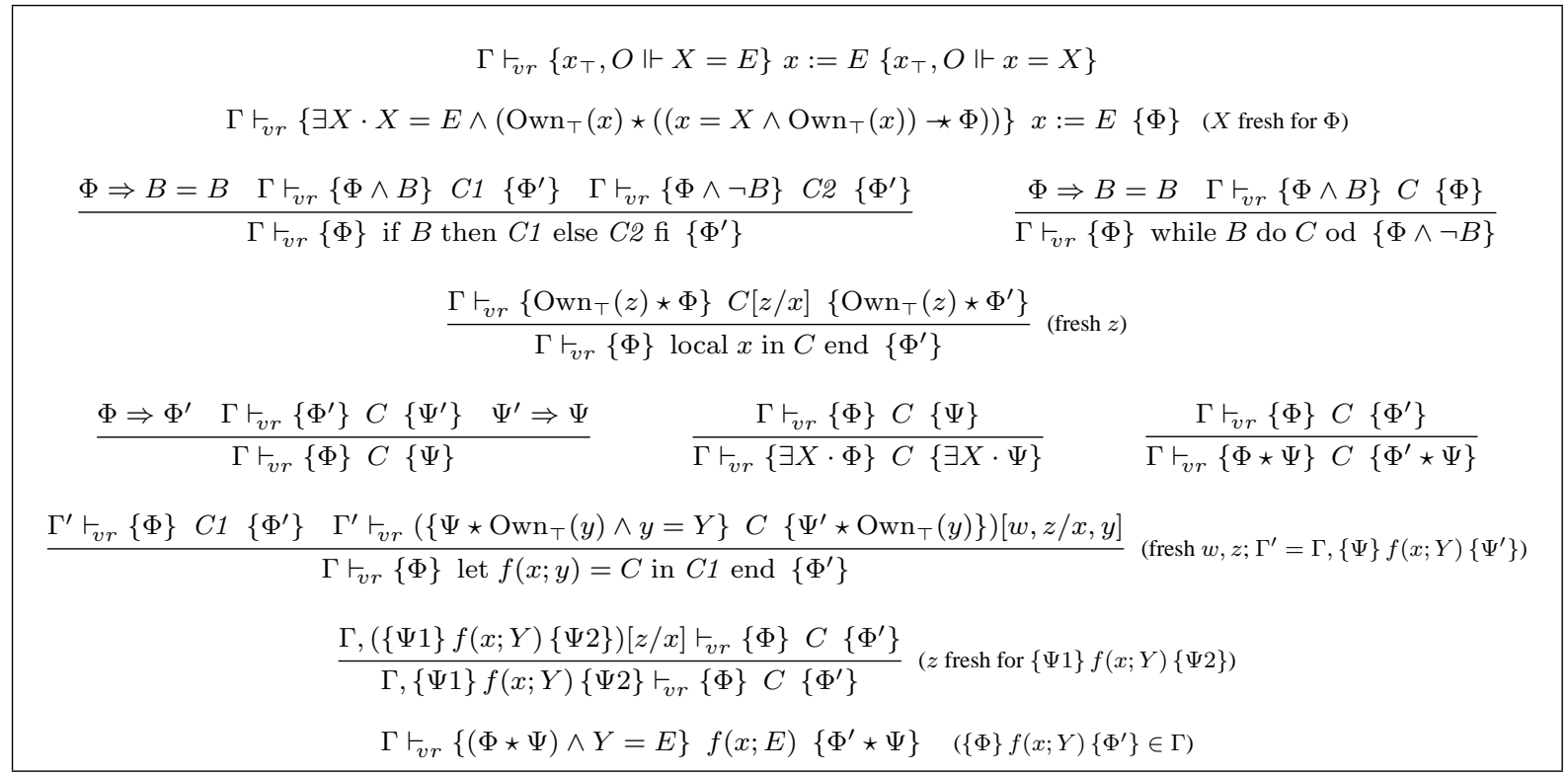

\section{Lemma 6.}

$$
\begin{aligned}
& (z \leftrightarrow x) s \stackrel{C[z / x]}{\longrightarrow} \rho_{\rho}^{n}(z \leftrightarrow x) s^{\prime} \quad \Rightarrow \quad s \stackrel{C}{\longrightarrow}_{\rho}^{n} s^{\prime} \\
& (z \leftrightarrow x) s \stackrel{C[z / x]}{\longrightarrow}{ }_{\rho}^{n} \text { unsafe } \Longleftrightarrow s \stackrel{C}{\longrightarrow}_{\rho}^{n} \text { unsafe } \\
& \text { ( } z \text { fresh for } C \text { and } \rho, x \notin \operatorname{dom}(s) \text { ). }
\end{aligned}
$$

Proof. By induction on the evaluation rules.

Lemma 7 (Determinacy). If $s \stackrel{C}{\longrightarrow} \rho \rho$ s1 and $s \stackrel{C}{\longrightarrow} \rho$ s2 then $s 1=$ s2.

Proof. By induction on the evaluation rules. The rules for local require lemma 6 . Other rules hold trivially.

In the semantics of triples, the precondition implies a safe computation, in contrast to the semantics of standard Hoare logic.

\section{Definition 8.}

$$
\begin{aligned}
& \rho \vDash_{n}\{\Phi\} C\left\{\Phi^{\prime}\right\} \stackrel{\text { def }}{=} \forall s, s^{\prime}, i . \\
& \left((s, i) \vDash \Phi \Rightarrow\left(\begin{array}{l}
s \stackrel{C}{\longrightarrow} \stackrel{n}{\rightarrow} \text { safe } \wedge \\
\left(s \stackrel{C}{\longrightarrow}{ }_{\rho}^{n} s^{\prime} \Rightarrow\left(s^{\prime}, i\right) \vDash \Phi^{\prime}\right.
\end{array}\right)\right)
\end{aligned}
$$

\section{Definition 9.}

$$
\rho \vDash_{n} \Gamma \stackrel{\text { def }}{=} \text { for every }\{\Phi\} f(x ; Y)\left\{\Phi^{\prime}\right\} \text { in } \Gamma,\left\langle f,\left(x^{\prime}, y, C\right)\right\rangle
$$
is in $\rho$ such that, for fresh $z$ and $w$,

$$
\rho \vDash_{n}\left(\begin{array}{c}
\left\{\Phi \star\left(y_{\top} \Vdash y=X\right)\right\} \\
C\left[x / x^{\prime}\right] \\
\left\{\Phi^{\prime} \star \mathrm{Own}_{\top}(y)\right\}
\end{array}\right)[z, w / x, y]
$$

Definition 10 (Semantics of judgements).

$$
\begin{aligned}
& \Gamma \vDash_{n}\{\Phi\} C\left\{\Phi^{\prime}\right\} \stackrel{\text { def }}{=} \\
& \quad \forall \rho \cdot\left(\left(\rho \vDash_{n} \Gamma\right) \Rightarrow\left(\rho \vDash_{n+1}\{\Phi\} C\left\{\Phi^{\prime}\right\}\right)\right)
\end{aligned}
$$

Theorem 11. If $\Gamma \vdash_{v r}\{\Phi\} C\left\{\Phi^{\prime}\right\}$ is derivable then $\forall n \cdot\left(\Gamma \vDash_{n}\{\Phi\} C\left\{\Phi^{\prime}\right\}\right)$

Proof. By induction on the derivation.

\section{Substitution}

In Hoare logic substitution is used to model assignment and parameter passing, but simple properties of substitution do not hold in our logic. In particular, substitution of formulae can affect ownership. $X=E \wedge \Phi \Rightarrow \Phi[E / X]$, for example, is not a tautology. (Here is a counter-example:

$$
\begin{gathered}
X=E \wedge\left(\left(X=X \wedge \mathbf{e m p}_{\mathbf{s}}\right) \star E=E\right) \\
\left.\Rightarrow \nRightarrow\left(E=E \wedge \mathbf{e m p}_{\mathbf{s}}\right) \star E=E\right)
\end{gathered}
$$

- the left side of the implication is satisfiable, while the right is false if $E$ contains program variables.) In the rest of this section we consider a subset of the logic in which substitution is well-behaved. As a result, we derive an assignment axiom that uses substitution.

A stack-imprecise formula does not notice extension of the stack and does not care about the quantity of permission it has for any variable.

Definition 12. $\Phi$ is stack imprecise $\stackrel{\text { def }}{=}$ $\forall s, s^{\prime}, i$.

$$
\left(((s, i) \vDash \Phi) \wedge\lfloor s\rfloor \subseteq\left\lfloor s^{\prime}\right\rfloor \Rightarrow\left(\left(s^{\prime}, i\right) \vDash \Phi\right)\right)
$$


Table 3. Operational semantics $s \stackrel{C}{\longrightarrow}{ }_{\rho}^{n} s^{\prime}$ and $s \stackrel{C}{\longrightarrow}{ }_{\rho}^{n}$ unsafe

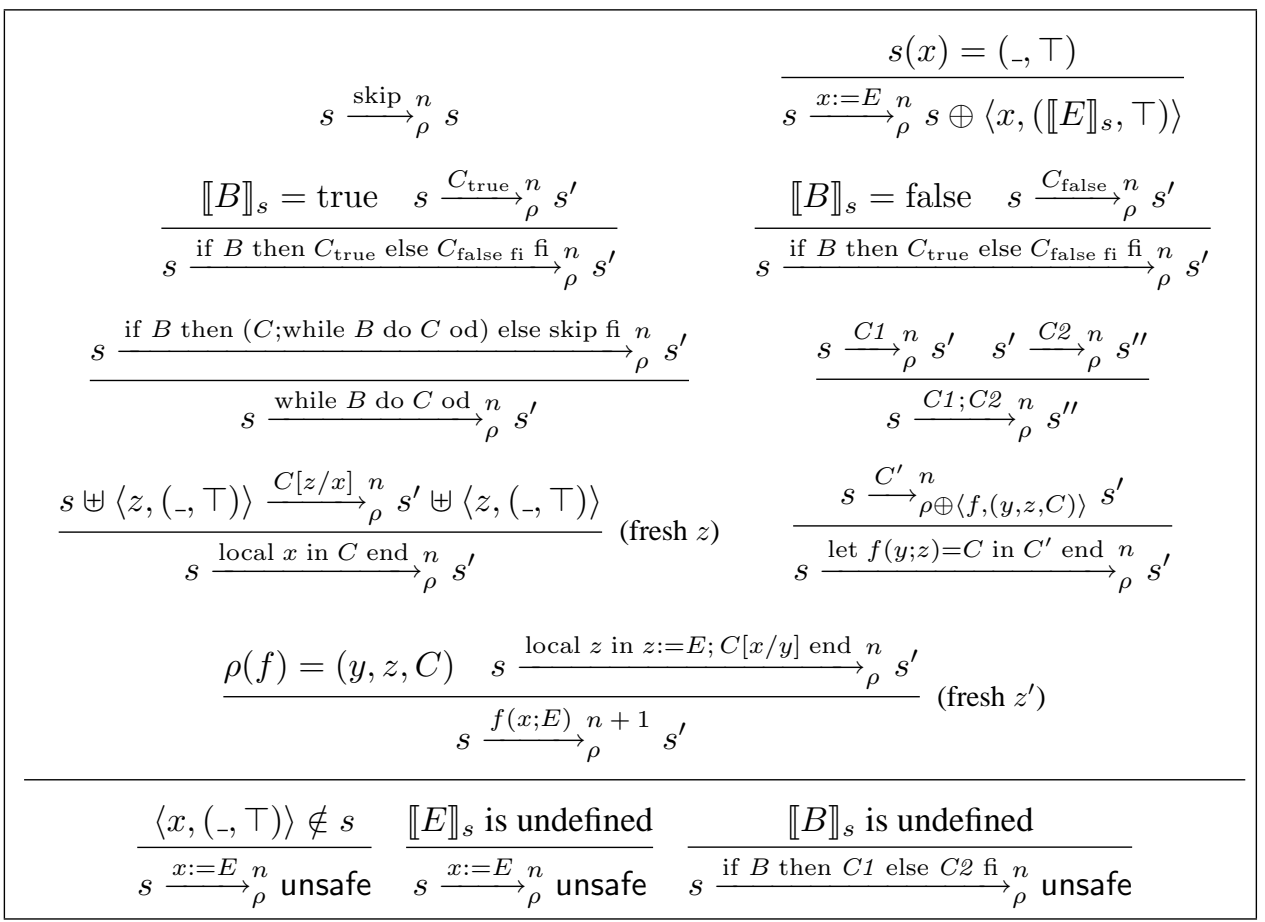

where $\lfloor s\rfloor=\{\langle x, v\rangle \mid\langle x,(v, p)\rangle \in s\}$

Lemma 13. If $\Phi$ and $\Psi$ are stack imprecise, then $\vDash \Phi \star \Psi \Leftrightarrow \Phi \wedge \Psi$

Corollary 14. If $\Phi$ is stack imprecise, then

$\vDash \Phi \star E=E^{\prime} \Leftrightarrow \Phi \wedge E=E^{\prime}$

We define implication in the same way as when intuitionistic implication is encoded into classical separation logic [10].

Definition 15 (Stack-imprecise $\Rightarrow$ and $\neg$ ).

$\Phi \stackrel{s}{\Rightarrow} \Phi^{\prime} \stackrel{\text { def }}{=}$ true $\star\left(\Phi \Rightarrow \Phi^{\prime}\right)$ and $\stackrel{s}{\neg} \Phi \stackrel{\text { def }}{=} \Phi \stackrel{s}{\Rightarrow}$ false.

Note: $E \neq E^{\prime} \Longleftrightarrow \stackrel{s}{\neg}\left(E=E^{\prime}\right)$ is a tautology.

If we restrict the syntax of formulae our logic can use substitution of equals for equals.

Definition 16 (restricted formulae).

$$
\begin{gathered}
\phi::=E=E|\phi \wedge \phi| \phi \vee \phi|\phi \stackrel{s}{\Rightarrow} \phi| \phi \star \star \phi|\pi=\pi| \\
\phi \star \phi|\forall X . \phi| \exists X . \phi \mid \text { false } \mid \text { true } \mid \stackrel{s}{\neg} \phi
\end{gathered}
$$

Lemma 17. Restricted formulae are stack imprecise.

Proof. Structural induction on $\phi$.

\section{Lemma 18.}

$(s, i) \vDash X=E \quad \Rightarrow \quad \llbracket E^{\prime} \rrbracket_{(s, i)}=\llbracket E^{\prime}[E / X] \rrbracket_{(s, i)}$

Proof. By induction on structure of $E^{\prime}$
Lemma 19. $\vDash X=E \Rightarrow(\phi \Leftrightarrow \phi[E / X])$

Proof. By structural induction on $\phi$. The $(\star)$ and $(-\star)$ cases require lemma 14 , and the $(=)$ case requires lemma 18 .

Definition 20. $\operatorname{vars}(O) \stackrel{\text { def }}{=}\left\{x \mid(x)_{p} \in O\right\}$

Lemma 21. ( O1 $\Vdash \phi 1) \star(O 2 \Vdash \phi 2) \Rightarrow(01,02 \Vdash$ $\phi 1 \star \phi 1)$

Lemma 22. If $\mathrm{FV}(\phi 1) \subseteq \operatorname{vars}(O 1)$ and $\mathrm{FV}(\phi 2) \subseteq$ $\operatorname{vars}($ O2) and $\vDash O \Vdash$ true $\Longleftrightarrow O 1 \Vdash$ true $\star O 2 \Vdash$ true then $\vDash(O \Vdash \phi 1 \star \phi 2) \Rightarrow(O 1 \Vdash \phi 1) \star(O 2 \Vdash \phi 2)$.

Lemma 23. $\vDash\left(\bar{y}_{\bar{\pi}} \Vdash \phi \star \psi\right) \Longleftrightarrow \exists \overline{p 1}, \overline{p 2} \cdot\left(\left(\bar{y}_{\overline{p 1}} \Vdash\right.\right.$ $\left.\phi) \star\left(\bar{y}_{\overline{p 1}} \Vdash \psi\right)\right) \wedge(\bar{\pi}=\overline{p 1} \circledast \overline{p 2})$

Theorem 24 (Assignment by substitution). $\Gamma \vdash_{v r}$ $\left\{x_{\top}, O \Vdash \phi[E / x] \wedge E=E\right\} \quad x:=E\left\{x_{\top}, O \Vdash \phi\right\}$ is derivable 
Proof.

$$
\begin{aligned}
& \left\{x_{\top}, \bar{y}_{\overline{p 1}} \Vdash E=X\right\} x:=E\left\{x_{\top}, \bar{y}_{\bar{p} 1} \Vdash x=X\right\} \\
& \begin{array}{c}
\left\{\left(x_{\top}, \bar{y}_{\overline{p 1}} \Vdash E=X\right) \star\left(\begin{array}{c}
\left(\bar{y}_{\overline{p 2}} \Vdash \phi[X / x]\right) \\
\wedge(\overline{p 1} \circledast \overline{p 1}=\bar{\pi})
\end{array}\right)\right\} \\
x:=E
\end{array} \\
& \left\{\left(x_{\top}, \bar{y}_{\overline{p 1}} \Vdash x=X\right) \star\left(\begin{array}{c}
\left(\bar{y}_{\overline{p 2}} \Vdash \phi[X / x]\right) \\
\wedge(\overline{p 1} \circledast \overline{p 1}=\bar{\pi})
\end{array}\right)\right\} \\
& \left\{\exists \overline{p 1}, \overline{p 2} \cdot\left(x_{\top}, \bar{y}_{\overline{p 1}} \Vdash E=X\right) \star\left(\begin{array}{c}
\left(\bar{y}_{\overline{p 2}} \Vdash \phi[X / x]\right) \\
\wedge(\overline{p 1} \circledast \overline{p 1}=\bar{\pi})
\end{array}\right)\right\} \\
& x:=E \\
& \left\{\exists \overline{p 1}, \overline{p 2} .\left(x_{\top}, \bar{y}_{\overline{p 1}} \Vdash x=X\right) \star\left(\begin{array}{c}
\left(\overline{\bar{y}_{\overline{p 2}}} \Vdash \phi[X / x]\right) \\
\wedge(\overline{p 1} \circledast \overline{p 1}=\bar{\pi})
\end{array}\right)\right\} \\
& \left\{x_{\top}, \bar{y}_{\bar{\pi}} \Vdash E=X \wedge \phi[E / x]\right\} x:=E\left\{x_{\top}, \bar{y}_{\bar{\pi}} \Vdash \phi\right\} \\
& \overline{\left\{\exists X \cdot x_{\top}, \bar{y}_{\bar{\pi}} \Vdash E=X \wedge \phi[E / x]\right\} x:=E\left\{\exists X \cdot x_{\top}, \bar{y}_{\bar{\pi}} \Vdash \phi\right\}} \\
& \left\{x_{\top}, \bar{y}_{\bar{\pi}} \Vdash E=E \wedge \phi[E / x]\right\} x:=E\left\{x_{\top}, \bar{y}_{\bar{\pi}} \Vdash \phi\right\}
\end{aligned}
$$

The first use of the rule of consequence requires

$$
\begin{aligned}
& x_{\top}, \bar{y}_{\bar{\pi}} \Vdash E=X \wedge \phi[E / x] \\
& \Rightarrow x_{\mathrm{T}}, \bar{y}_{\bar{\pi}} \Vdash E=X \wedge \phi[X / x][E / X] \\
& \Rightarrow x_{\top}, \bar{y}_{\bar{\pi}} \Vdash(E=X) \star \phi[X / x] \\
& \Rightarrow \exists p, p^{\prime}, \overline{p 1}, \overline{p 2} \text {. } \\
& \left(\left(x_{p}, \bar{y}_{\overline{p 1}} \Vdash E=X\right) \star\left(x_{p^{\prime}}, \bar{y}_{\overline{p 2}} \Vdash \phi[X / x]\right)\right) \\
& \wedge\left(p \circledast p^{\prime}=\top\right) \wedge(\overline{p 1} \circledast \overline{p 2}=\bar{\pi}) \quad \text { (Lemma 23) } \\
& \Rightarrow \exists \overline{p 1}, \overline{p 2} .\left(\left(x_{\top}, \bar{y}_{\overline{p 1}} \Vdash E=X\right) \star\left(\bar{y}_{\overline{p 2}} \Vdash \phi[X / x]\right)\right) \\
& \wedge(\overline{p 1} \circledast \overline{p 2}=\bar{\pi}) \\
& \text { (Lemma 21) } \\
& \Rightarrow \exists \overline{p 1}, \overline{p 2} .\left(x_{\top}, \bar{y}_{\overline{p 1}} \Vdash E=X\right) \\
& \star\left(\left(\bar{y}_{\overline{p 2}} \Vdash \phi[X / x]\right) \wedge(\overline{p 1} \circledast \overline{p 2}=\bar{\pi})\right) \\
& \overline{p 2}=\bar{\pi}))
\end{aligned}
$$

and

$$
\begin{aligned}
& \exists \overline{p 1}, \overline{p 2} .\left(x_{\top}, \bar{y}_{\overline{p 1}} \Vdash x=X\right) \\
& \quad \star\left(\left(\bar{y}_{\overline{p 2}} \Vdash \phi[X / x]\right) \wedge(\overline{p 1} \circledast \overline{p 2}=\bar{\pi})\right) \\
& \Rightarrow x_{\top}, \bar{y}_{\bar{\pi}} \Vdash(x=X) \star \phi[X / x] \\
& \Rightarrow x_{\top}, \bar{y}_{\bar{\pi}} \Vdash x=X \wedge \phi[X / x] \\
& \Rightarrow x_{\top}, \bar{y}_{\bar{\pi}} \Vdash \phi
\end{aligned}
$$

The second use of the rule of consequence requires

$$
E=E \Rightarrow \exists X \cdot E=X
$$

\section{Encoding Hoare logics}

Our logic accepts translations of Hoare logic proofs. We present a translation of a Hoare logic with reference and value parameters in procedure definitions. We use $\phi$ and $\psi$ to range over Hoare logic assertions since there is an implicit translation to restricted formulae: $\stackrel{s}{\Rightarrow}$ for $\Rightarrow, \stackrel{s}{\neg}$ for $\neg$.

A Hoare-logic function context $\mathbb{F}$ (cf. $\Gamma$ ) is a set of function specifications $\{\phi\} f(x ; y)[\bar{u} ; \bar{v}]\{\psi\}$ where
- $f$ is a function name, $x$ a reference-parameter name and $y$ a value-parameter name;

- $\phi$ is the precondition and $\psi$ the postcondition of $f(x ; y)$;

- $\bar{u}$ is a set of the names of the global variables modifed by $f(x ; y)$ and $\bar{v}$ a set of the names of global variables it reads;

- $\bar{u} \subseteq \bar{v}$

Table 4 defines $\operatorname{mods}(\mathbb{F}, C)$, the variables written by $C$, and free $(\mathbb{F}, C)$, its free variables: because of the complexities of the let definition we require two definitions for function call but, because let declares functions one at a time, we do not need a fixed-point iteration. Table 5 gives the rules of the Hoare logic which we encode.

Lemma 25 (The logics are equivalent on defined assertions).

$$
\mathrm{FV}(\phi) \subseteq \operatorname{dom}(s) \Rightarrow\left((\lfloor s\rfloor, i) \vDash_{H} \phi \Longleftrightarrow(s, i) \vDash_{v r} \phi\right)
$$

Proof. Structural induction on $\phi$. The interesting case is $\star$, which requires that $\phi$ is stack imprecise.

Definition 26 (Supporting write and read variables).

$$
\operatorname{supports}_{\bar{p}}(\bar{u} ; \bar{v}) \stackrel{\text { def }}{=}\left(\bar{u}_{1}\right)_{\top}, \ldots,\left(\bar{u}_{m}\right)_{\top},\left(\bar{w}_{1}\right)_{\bar{p}_{1}}, \ldots,\left(\bar{w}_{n}\right)_{\bar{p}_{n}}
$$
where $\bar{w}=\bar{v} \backslash \bar{u}$

Definition 27 (Supporting a command).

$$
\operatorname{supports}_{\bar{p}}(\mathbb{F}, C) \stackrel{\text { def }}{=} \operatorname{supports}_{\bar{p}}(\operatorname{mods}(\mathbb{F}, C) ; \operatorname{free}(\mathbb{F}, C))
$$

In Hoare logic program variables and logical variables are conflated. In our translation of $\{\phi\} C\{\psi\}$ we turn all the free variables of $\phi$ and $\psi$ that are not used in $C$ into logical variables.

Definition 28 (Triple translation).

$$
\begin{gathered}
\llbracket\{\phi\} C\{\psi\} \rrbracket_{\mathbb{F}} \stackrel{\text { def }}{=}\{O \Vdash \phi[\bar{U} / \bar{u}]\} C\{O \Vdash \psi[\bar{U} / \bar{u}]\} \\
\text { where } O=\operatorname{supports}_{\bar{p}}(\mathbb{F}, C) ; \\
\bar{u}=\mathrm{FV}(\phi, \psi) \backslash \operatorname{vars}(O) ; \\
\text { fresh } \bar{p}, \bar{U}
\end{gathered}
$$

(Here $\bar{p}$ and $\bar{U}$ are sets of fresh logical variables, implicitly quantified at the level of the triple: that is, because of the semantics of triples, the fresh $\bar{p}, \bar{U}$ can be thought of as universally quantified.

$$
\forall \bar{p}, \bar{U} \cdot\{O \Vdash \phi[\bar{U} / \bar{u}]\} C\{O \Vdash \psi[\bar{U} / \bar{u}]\}
$$

Clearly, the translation is deterministic.) 
Table 4. Modified and free variables of commands $\operatorname{mods}(\mathbb{F}, C)$, free $(\mathbb{F}, C)$

\begin{tabular}{|c|c|c|}
\hline$C$ & $\operatorname{mods}(\mathbb{F}, C)$ & free $(\mathbb{F}, C)$ \\
\hline$x:=E$ & $\{x\}$ & $\mathrm{FV}(E) \cup\{x\}$ \\
$C_{1} ; C_{2}$ & $\operatorname{mods}\left(\mathbb{F}, C_{1}\right) \cup \operatorname{mods}\left(\mathbb{F}, C_{2}\right)$ & $\operatorname{free}\left(\mathbb{F}, C_{1}\right) \cup$ free $\left(\mathbb{F}, C_{2}\right)$ \\
skip & $\varnothing$ & $\varnothing$ \\
while $B$ do $C$ od & $\operatorname{mods}(\mathbb{F}, C)$ & $\mathrm{FV}(B) \cup$ free $(\mathbb{F}, C)$ \\
local $x$ in $C$ end & $\operatorname{mods}(\mathbb{F}, C) \backslash\{x\}$ & free $(\mathbb{F}, C) \backslash\{x\}$ \\
if $B$ then $C_{1}$ else $C_{2}$ fi & $\operatorname{mods}\left(\mathbb{F}, C_{1}\right) \cup \operatorname{mods}\left(\mathbb{F}, C_{2}\right)$ & $\mathrm{FV}(B) \cup$ free $\left(\mathbb{F}, C_{1}\right) \cup$ free $\left(\mathbb{F}, C_{2}\right)$ \\
let $f(x ; y)=C$ in $C^{\prime}$ end & & \\
where $\mathbb{F}^{\prime}=\mathbb{F},\{-\} f(x ; y)[\bar{u} ; \bar{v}]\{-\} ;$ & $\operatorname{mods}\left(\mathbb{F}^{\prime}, C^{\prime}\right)$ & \\
$\bar{u}=\operatorname{mods}(\mathbb{F}, C) \backslash\{x, y\} ;$ & & \\
$\bar{v}=$ free $(\mathbb{F}, C) \backslash\{x, y\}$ & $\{x\} \cup \bar{u}$ & $\{x\} \cup \mathrm{FV}(E) \cup \bar{v}$ \\
$f(x ; E)$ (normal case, $\{-\} f(x ; y)[\bar{u} ; \bar{v}]\{-\} \in \mathbb{F})$ & $\{x\}$ & $\{x\} \cup \mathrm{FV}(E)$ \\
$f(x ; E)$ (bootstrap case, $\{-\} f(-;-)[-;]\{-\} \notin \mathbb{F})$ & & \\
\hline
\end{tabular}

Table 5. Hoare logic rules: $\quad \mathbb{F} \vdash_{H}\{\phi\} C\{\phi\}$

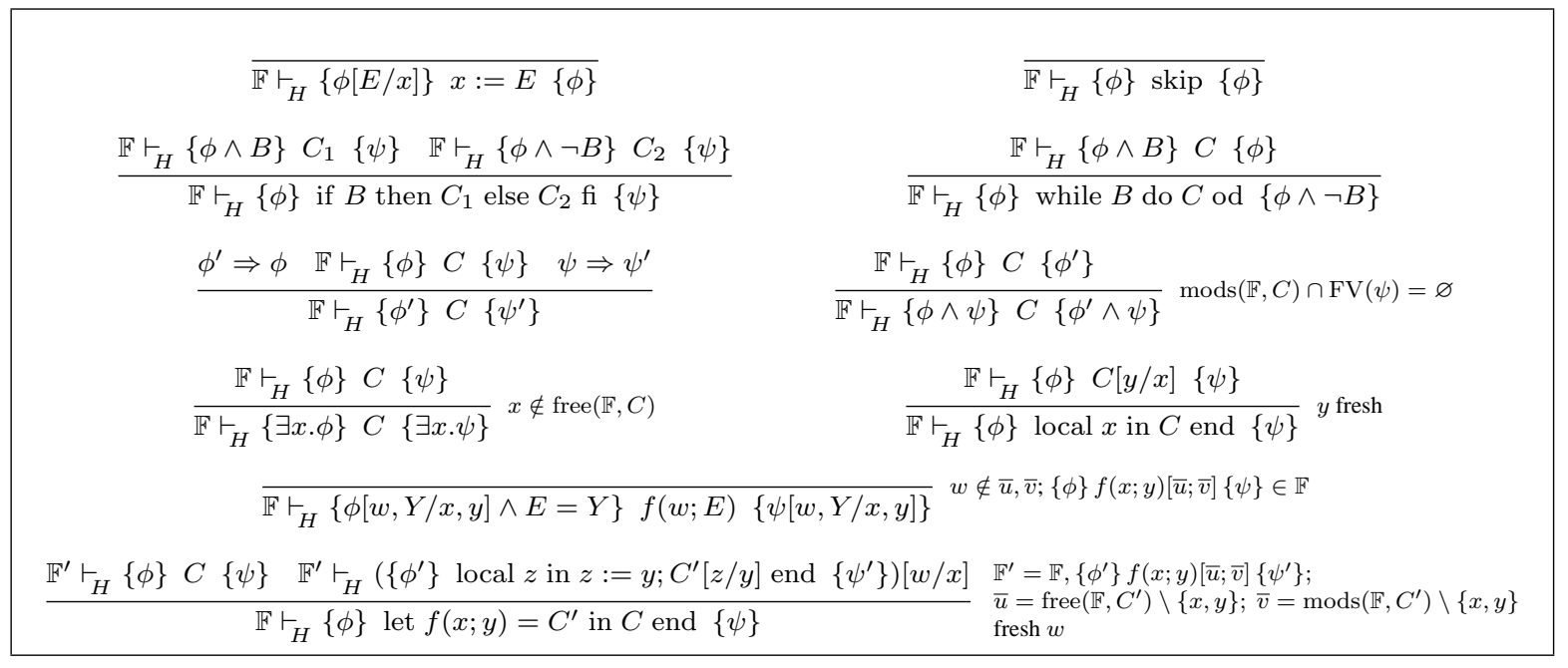

Although our translation replaces some integer program variables with new logical variables, we can always retrieve the original specification by extending $O$ and using the frame rule to enforce an invariant which equates the values of new and old variables.

Lemma 29 (From proof with logical variables infer proof with program variables.).

Proof.

$$
\begin{aligned}
& \{O \Vdash \phi[Z / z]\} C\{O \Vdash \psi[Z / z]\} \\
& \overline{\left\{\begin{array}{c}
\left(z_{\pi} \Vdash Z=z\right) \\
\star(O \Vdash \phi[Z / z])
\end{array}\right\} C\left\{\begin{array}{c}
\left(z_{\pi} \Vdash Z=z\right) \\
\star(O \Vdash \psi
\end{array}\right\}} \\
& \frac{\left\{\exists Z \cdot\left(\begin{array}{c}
\left(z_{\pi} \Vdash Z=z\right) \\
\star(O \Vdash \phi[Z / z])
\end{array}\right)\right\} C\left\{\exists Z \cdot\left(\begin{array}{c}
\left(z_{\pi} \Vdash Z=z\right) \\
\star(O \Vdash \psi[Z / z])
\end{array}\right)\right\}}{\left\{O, z_{\pi} \Vdash \phi\right\} C\left\{O, z_{\pi} \Vdash \psi\right\}}
\end{aligned}
$$

Definition 30 (Translation of procedure environment).

$\llbracket \mathbb{F} \rrbracket \stackrel{\text { def }}{=}\left\{\llbracket \begin{array}{c}\{\phi[Y / y]\} \\ f(x ; Y) \\ \{\psi[Y / y]\}\end{array} \|_{\mathbb{F}} \mid\{\phi\} f(x ; y)[\bar{u} ; \bar{v}]\{\phi\} \in \mathbb{F}\right\}$

Note: For convenience, we assume that triple translation treats $Y$ in $f(x ; Y)$ as a constant.

Theorem 31 (Completeness of encoding).

$$
\left(\mathbb{F} \vdash_{H}\{\phi\} C\{\psi\}\right) \Rightarrow\left(\llbracket \mathbb{F} \rrbracket \vdash_{v r} \llbracket\{\phi\} C\{\psi\} \rrbracket_{\mathbb{F}}\right)
$$

Proof. By induction on the Hoare-logic derivation.

Theorem 32 (Soundness of encoding).

$$
\left(\llbracket \mathbb{F} \rrbracket \vDash_{v r} \llbracket\{\phi\} C\{\psi\} \rrbracket_{\mathbb{F}}\right) \Rightarrow\left(\mathbb{F} \vDash_{H}\{\phi\} C\{\psi\}\right)
$$


Proof.

$$
\begin{aligned}
&\left(\mathbb{F} \vDash_{v r} \llbracket\{\phi\} C\{\psi\} \rrbracket_{\mathbb{F}}\right) \\
& \Rightarrow\left(\mathbb{F} \vDash_{v r}\left\{O, O^{\prime} \Vdash \phi\right\} C\left\{O, O^{\prime} \Vdash \psi\right\}\right)
\end{aligned}
$$

where $O=\operatorname{supports}_{\bar{p}}(\mathbb{F}, C)$, and $\operatorname{vars}\left(O, O^{\prime}\right) \supseteq$ $\mathrm{FV}(\phi, \psi)$, follows directly from repeated application of lemma 29. Then

$$
\begin{aligned}
\left(\mathbb{F} \vDash_{v r}\left\{O, O^{\prime} \Vdash \phi\right\} C\left\{O, O^{\prime} \Vdash \psi\right\}\right) \\
\Rightarrow\left(\mathbb{F} \vDash_{H}\{\phi\} C\{\psi\}\right)
\end{aligned}
$$

follows directly from lemma 25.

\section{Concurrency}

Hoare-logic concurrency rules have complex side conditions and restrictions constraining the use of variables. In our logic we do not need any of that. The rules are given with respect to a resource context, $\Delta$, which maps a resource identifier $b$ to its corresponding invariant. The invariants must be precise [5]. Table 6 gives the rules.

\subsection{Soundness}

Brookes has shown this logic to be sound [4].

\subsection{Translation}

We can translate Brookes's rules for concurrent separation logic [5] into our own (we omit his treatment of the heap). The key to his soundness proof is the notion of critical variable. A variable is critical if it is modified in one thread and free in another: in $x:=y \| y:=z$, for example, $y$ is critical.

Each critical variable is associated with a resource and each access must be within a critical region for that resource. A resource context $\mathbb{R}$ maps a resource name $b$ to a critical-variable list $\bar{u}$ and an invariant $\psi$. There are two operations on these contexts: (1) $\operatorname{crit}(\mathbb{R})$ delivers the critical variables in $\mathbb{R}$; and $(2) \mathrm{FV}(\mathbb{R})$ the variables free in the invariants as well as all critical variables. Brookes's parallel rule has a side condition:

$$
\begin{aligned}
& \mathrm{FV}\left(\Phi 1, \Phi 1^{\prime}\right) \cap \operatorname{mods}(C 2)=\varnothing \\
& \mathrm{FV}\left(\Phi 2, \Phi 2^{\prime}\right) \cap \operatorname{mods}(C 1)=\varnothing \\
& \mathrm{FV}(C 1) \cap \operatorname{mods}(C 2) \subseteq \operatorname{crit}(\mathbb{R}) \\
& \mathrm{FV}(C 2) \cap \operatorname{mods}(C 1) \subseteq \operatorname{crit}(\mathbb{R})
\end{aligned}
$$

The $x:=E$ rule has the side condition $x \notin \mathrm{FV}(\mathbb{R})$. There is also an additional constraint on the well-formedness of judgements $\mathbb{R} \vdash_{H}\{\Phi\} C\left\{\Phi^{\prime}\right\}$ : the critical variables may not be mentioned in the pre- or post-condition, i.e. $\operatorname{crit}(\mathbb{R}) \cap \mathrm{FV}\left(\Phi, \Phi^{\prime}\right)=\varnothing$.

\author{
Definition 33. \\ $\operatorname{supports}_{\bar{p}}(\mathbb{R}, C) \stackrel{\text { def }}{=}$ \\ $\operatorname{supports}_{\bar{p}}((\operatorname{mods}(C) \backslash \mathrm{FV}(\mathbb{R})) ;(\operatorname{free}(C) \backslash \operatorname{crit}(\mathbb{R})))$
}

Definition 34 (Triple translation).

$\llbracket\{\phi\} C\{\psi\} \rrbracket_{\mathbb{R}} \stackrel{\text { def }}{=}\{O \Vdash \phi[\bar{U} / \bar{u}]\} C\{O \Vdash \psi[\bar{U} / \bar{u}]\}$ where $O=\operatorname{supports}_{\bar{p}}(\mathbb{R}, C), \bar{u}=\mathrm{FV}(\phi, \psi) \backslash \operatorname{vars}(O)$, and $\bar{p}, \bar{U}$ are sets of fresh logical variables.

Definition 35 (Context translation, $\llbracket \mathbb{R} \rrbracket$ ). We translate each element of the context to

$$
\llbracket r[\bar{u}]: \psi \rrbracket=r: \operatorname{supports}_{\bar{p}}(\bar{u}, \mathrm{FV}(\psi)) \Vdash \psi
$$

\section{Lemma 36.}

$$
\begin{array}{r}
\operatorname{supports}_{\bar{p}}(\mathbb{R}, C) \star \operatorname{supports}_{\bar{p}}(\bar{u}, \mathrm{FV}(\psi)) \\
=\operatorname{supports}_{\bar{p}}(\mathbb{R} \uplus\langle b,(\bar{u}, \psi)\rangle, C)
\end{array}
$$

Theorem 37 (Completeness of encoding).

$$
\mathbb{R} \vdash_{H}\{\phi\} C\{\psi\} \quad \Rightarrow \quad \llbracket \mathbb{R} \rrbracket \vdash_{v r} \llbracket\{\phi\} C\{\psi\} \rrbracket_{\mathbb{R}}
$$

Proof. By induction on the derivation for Brookes's rules.

Theorem 38 (Soundness of encoding).

Proof. Same as theorem 32

\section{Conclusions}

We have a logic which admits translations of all Hoare logic proofs and in which there are no side conditions on the use of variables or restrictions on the action of concurrent programs. In addition we are able (though not within the space constraints of this presentation) to deal with the heap in the same way. By working through several examples, Bornat has previously shown that this kind of logic deals conveniently with the verification of shared-variable concurrency programs [3]. His logic can be translated into our own, and its soundness is a consequence of the soundness of our own.

In all other previous Hoare logics a simultaneous treatment of concurrency, procedure call and the heap requires complex side conditions on the use of variables, as well as restrictions on the action of programs which are extremely difficult to check in a mechanical proof tool. Smallfoot [1], for example, uses a treatment of concurrency based on Brookes' and O'Hearn's treatment in separation logic, and must make a completely global static analysis when dealing with the restrictions on concurrent programs. The analysis 
Table 6. Variables as resource rules for concurrency

$$
\begin{gathered}
\frac{\Delta \vdash_{v r}\{\Phi 1\} C 1\left\{\Phi 1^{\prime}\right\} \quad \Delta \vdash_{v r}\{\Phi 2\} C 2\left\{\Phi 2^{\prime}\right\}}{\Delta \vdash_{v r}\{\Phi 1 \star \Phi 2\} C 1 \| C 2\left\{\Phi 1^{\prime} \star \Phi 2^{\prime}\right\}} \frac{\Delta, b: \Psi \vdash_{v r}\{\Phi\} C\left\{\Phi^{\prime}\right\}}{\Delta \vdash_{v r}\{\Phi \star \Psi\} \text { resource } b \text { in } C \text { end }\left\{\Phi^{\prime} \star \Psi\right\}} \\
\frac{\Delta \vdash_{v r}\{(\Phi \star \Psi) \wedge B\} C\left\{\Phi^{\prime} \star \Psi\right\} \quad \Phi \star \Psi \Rightarrow B=B}{\Delta, b: \Psi \vdash_{v r}\{\Phi\} \text { with } b \text { when } B \operatorname{do} C \text { od }\left\{\Phi^{\prime}\right\}}
\end{gathered}
$$

takes several pages to describe, and is extremely intricate to implement. No such analysis would be required in a tool based on our new logic.

\section{Acknowledgements}

Like much of our previous work in program logic, this paper emerges from repeated rumbustious discussions within the East London Massive, a frequent but irregular gathering at Queen Mary, University of London. We acknowledge in particular the seminal contribution of Peter O'Hearn in proposing that we undertake this work and then attempting to trip us up at ever turn, right up to the very last. Hongseok Yang, from outside the Massive, provided a model for Bornat's logic and inspired us towards the elimination of all side conditions in our own work.

The landlord of L'Oasis in the Mile End Road fed us well before every Massive meeting. Parkinson and Bornat are grateful to WAGN plc for the several occasions on which they ran to time over the last few months, and hope for several more surprises of the same kind before the well-deserved, long-awaited and surely now inevitable renationalisation of all British railways.

This work was supported by EPSRC grants EP/C523997/1 (Parkinson and Bornat) and EP/C544757/1 (Calcagno). Parkinson and Bornat also thank Intel Research Cambridge for their support.

\section{References}

[1] J. Berdine, C. Calcagno, and P. W. O'Hearn. Modular automatic assertion checking with separation logic. Draft, Nov. 2005.

[2] R. Bornat, C. Calcagno, P. O'Hearn, and M. Parkinson. Permission accounting in separation logic. In POPL '05: Proceedings of the 32nd ACM SIGPLAN-SIGACT sysposium on Principles of programming languages, pages 259-270, New York, NY, USA, Jan. 2005. ACM Press.

[3] R. Bornat, C. Calcagno, and H. Yang. Variables as resource in separation logic. Presented at MFPS XXI, Birmingham, May 2005. To appear in Electronic Notes in Computer Science, 2005.

[4] S. Brookes. Variables as resource for shared-memory programs: Semantics and soundness. In Proceedings of MFPS XXII. Elsevier ENTCS., May 2006.
[5] S. D. Brookes. A semantics for concurrent separation logic. In CONCUR'04: 15th International Conference on Concurrency Theory, volume 3170 of Lecture Notes in Computer Science, pages 16-34, London, Aug. 2004. Springer. Extended version to appear in Theoretical Computer Science.

[6] R. Cartwright and D. Oppen. Unrestricted procedure calls in hoare's logic. In POPL '78: Proceedings of the 5th ACM SIGACT-SIGPLAN symposium on Principles of programming languages, pages 131-140, New York, 1978. ACM Press.

[7] D. Gries and G. Levin. Assignment and procedure call proof rules. ACM Transactions on Programming Languages and Systems, 2(4), Oct. 1980.

[8] C. A. R. Hoare. An axiomatic basis for computer programming. Commun. ACM, 12(10):576-580, 1969.

[9] C. A. R. Hoare. Towards a theory of parallel programming. Operating Systems Techniques, 1971.

[10] S. S. Ishtiaq and P. W. O'Hearn. BI as an assertion language for mutable data structures. In Symposium on Principles of Programming Languages, pages 14-26, 2001.

[11] P. O'Hearn, J. Reynolds, and H. Yang. Local reasoning about programs that alter data structures. In L. Fribourg, editor, CSL 2001, pages 1-19. Springer-Verlag, 2001. LNCS 2142.

[12] P. W. O'Hearn. Resources, concurrency and local reasoning. To appear in Theoretical Computer Science; preliminary version published as [13].

[13] P. W. O'Hearn. Resources, concurrency and local reasoning. In CONCUR'04: 15th International Conference on Concurrency Theory, volume 3170 of Lecture Notes in Computer Science, pages 49-67, London, Aug. 2004. Springer. Extended version is [12].

[14] S. Owicki and D. Gries. An axiomatic proof technique for parallel programs. Acta Informatica, 19:319-340, 1976.

[15] J. C. Reynolds. Intuitionistic reasoning about shared mutable data structure. In J. Davies, B. Roscoe, and J. Woodcock, editors, Millennial Perspectives in Computer Science, pages 303-321. Palgrave, 2000.

[16] J. C. Reynolds. Separation logic: A logic for shared mutable data structures. In LICS '02: Proceedings of the 17th Annual IEEE Symposium on Logic in Computer Science, pages 5574, Washington, DC, USA, 2002. IEEE Computer Society.

[17] C. Strachey. Fundamental concepts in programming languages. Higher Order Symbolic Computation, 13(1-2):1149, 2000. 